\title{
LETTERS
}

\section{The hundred-dollar genome: a health care cart before the genomic horse}

The science of genetics is grounded in the principles of inheritance outlined over 150 years ago. Almost 100 years later, the physical bases for these principles were articulated in the DNA model published in 1953 by Watson and Crick. ${ }^{1}$ Together, they provided a framework to explain transmission of like begets like and accommodated incredible differences seen across life forms, as well as across individuals within a species. In doing so, they provided the very foundation for the universality of evolution by natural selection and unified all life sciences.

The next stepping stone in advancing genetic research came with the technological developments that allowed direct appraisal of DNA leading to the sequencing of the complete genomes and the current "omics" revolution. Now, it is possible to assess an individual for their complete set of DNA, RNA and proteins, including some of the regulatory features. Finally, we are able to directly look at the genome and explore such fundamental questions as, where does it come from? What does it do? How does it do it, and what may go wrong? The secret of life is being divulged in increasing detail.

Probably the most important development in advancing genetic research came with making DNA technologies affordable for individual researchers across the world. For example, the human genome project that produced a composite of the human genome sequence was completed at a cost of US\$2.7 billion in 1991 dollars. Assembly of the complete genome sequence of an individual human at the cost of US\$100 million dollars followed in 2007. Ten years later, an individual genome sequence is being assembled at a cost of US\$1000, and we are not done yet. Soon, it may cost US\$100 to sequence the complete genome of any human, whether newborn, young or old. It will be a permanent and most comprehensive descrip- tion of the individual. As it stands, the interpretation of the results will be most challenging. Yet, they will hold the key to the past, present and future. It just keeps on getting cheaper and better.

This affordability and utmost resolution are allowing researchers to consider research that would have been unthinkable just a few years ago. It is not uncommon to use the complete genome sequence of patient(s) with a disease in relation to their unaffected relatives while assessing connection of gene mutation(s) causing the disease. Interestingly, this research has moved the genome sequence to include variations in the general population. The projects on sequencing genomes of normally functioning individuals from the general population cover all DNA sequence differences or variants that may have positive, negative or no effect on our daily lives. Some of these effects are known, whereas others are viewed as variants of unknown significance. More important, the genome sequence data could be stored, archived and reanalyzed at timely intervals using ever-improving tools, technologies and understanding of human variations. We have moved to a new era of prediction of health and well-being. The question is, are we ready for it?

Comparisons of individual genomes representing the general population have begun to generate novel insights and open new directions to questions that were beyond the bounds of any inquiry just a year ago. The research study on the first 56 participants in the Personal Genome Project Canada, published in CMAJ, reported that "most variants were of uncertain significance or likely benign," "most participants (53/56) carried at least 1 disease-associated allele" and, in about $25 \%$ of individuals, the whole genome sequencing can benefit their routine health care in the future. ${ }^{2}$ The implication and interpretation of such results will continue to be challenging.

In the future, it is likely that genome sequences of individuals will be generated. In some instances, the genome sequence will be generated to guide health and well-being issues for life that may include early diagnosis and personalized medicine; whereas, for others, it will represent a curiosity, not unlike what is available currently through a variety of ancestry-related commercial operations. The generation of genome sequences will be irresistible at a cost of $\$ 100$. There is no doubt that they will be generated on a large scale, because we can and with no obvious purpose. Yet, every personal genome sequence will carry with it a variety of health and societal implications. It will bring about novel ethical questions that should not be left to a single individual or group to identify and offer solutions. It deserves broad societal input.

What is needed is for health professionals and the general public to become informed, interested and involved. The public must take full ownership of this issue. The $\$ 100$ genome is here to stay. Like a driver's licence and health card, it will represent a critical piece of identity. However, more than the driver's licence and health card, sharing of this information with others must be protected. To this end, legislation like the Genetic NonDiscrimination Act in Canada will be needed and necessary. There is a plethora of insights in the $\$ 100$ genome. Only the most careful application and sharing of this insight will assure its full value for the individual, his or her family and society.

\section{Shiva Singh PhD}

Professor, Department of Biology, Western University, London, Ont.

- Cite as: CMAJ 2018 April 23;190:E514. doi: $10.1503 / \mathrm{cmaj} .69259$

\section{References}

1. Watson JD, Crick FHC. Molecular structure of nucleic acids: a structure for deoxyribose nucleic acid. Nature 1953;171:737-8.

2. Reuter MS, Walker S, Thiruvahindrapuram B, et al. The Personal Genome Project Canada: findings from whole genome sequences of the inaugural 56 participants. CMAJ 2018;190:E126-36.

Competing interests: None declared. 